INVESTIGACIONES

\title{
DIMENSIONES DE FRACTURA COGNITIVA EN FORMACION INICIAL DOCENTE EN CHILE: UN ESTUDIO DE CASOS EN TRES CONTEXTOS FORMATIVOS*
}

\author{
Dimensions of cognitive fracture in initial teacher training in Chile:
}

A study of cases in three formative contexts

\section{Iván Oliva, Nicolás Díaz, Paulina Larrosa, Paulo Contreras, Christian Miranda}

\author{
Universidad Austral de Chile
}

\begin{abstract}
Resumen
El artículo expone los principales supuestos teóricos y metodológicos de un programa de investigación en complejidad y transdisciplina, orientado en este contexto específico a cartografiar nodos epistemológicos críticos en formación inicial docente (FID). El marco referencial de la investigación, como su prospectiva metodológica, se centra en tres ejes de fractura cognitiva descritos para la FID, a saber: desvinculación teoría/praxis, disyunción formativa disciplina/pedagogía e incomunicación entre los diversos dominios disciplinarios de formación pedagógica. Se exponen los principales núcleos interpretativos de un trabajo de campo en tres contextos institucionales de FID en Chile.
\end{abstract}

Palabras clave: epistemología, formación inicial docente, complejidad.

\section{Summary}

The article sets out the main theoretical and methodologic assumptions of a research program in complexity and transdiscipline, oriented against this specific background to map epistemologic critics nodes in initial teacher training (ITT). The referential frame of the investigation, like their prospective methodologic one, concentrated in three described axes of mental fracture for the EIF, that is to say: separation theory/praxis, formative disjunction discipline/pedagogy and isolation between the disciplinary dominions diversity of pedagogical formation. The main interpretative nuclei of a work of field in three institutional contexts of ITT in Chile are exposed.

Key words: epistemology, initial teacher training, complexity.

* El presente reporte de investigación se enmarca en una investigación financiada por la Dirección de Investigación y Desarrollo (DID) de la Universidad Austral de Chile. (S-2008-05) y en una aproximación de campo específica del proyecto FONDECYT (11080193). 


\section{ANTECEDENTES GENERALES DEL PROBLEMA}

El presente reporte se inscribe en una línea de investigación en complejidad y transdisciplina orientada, en este contexto específico, al cartografiado de dimensiones de fractura en los procesos de configuración de conocimiento en formación inicial docente (en adelante FID) en Chile. Desde esta base, todo proceso educativo supone configuraciones semióticas explícitas e implícitas, lo que da a la noción de paradigma una relevancia no solo epistemológica, sino además política, en tanto condición y posibilidad de las reformas educativas contemporáneas. De esta forma, una paradigmatología del fenómeno educativo es inherente a la tarea de indagar en las emergentes matrices epistémicas del sistema. El campo de indagación de la presente investigación se centra en diversos contextos disciplinares e institucionales de formación inicial docente en Chile, en tanto instancias neurálgicas en términos de calidad y equidad educativa (OCDE, 2009).

A partir de la convergencia de los elementos de la teoría de la complejidad, cibernética de segundo orden, ciencias cognitivas y ciencias de la educación, se desarrolla una aproximación metodológica a los fenómenos de configuración de conocimiento en educación, mediante la construcción, validación y análisis de redes cognitivas.

Holográficamente y a modo de una fractalidad semiótica, la educación en sus procesos de formación conlleva la presencia o huella matricial de la trama sociocultural a la que pertenece; recursivamente, la trama sociocultural genera y legitima la educación que la genera y legitima (Morin, 1999). Desde la configuración de este bucle, la problemática sociocultural se infiltra recursivamente en el fenómeno educativo e invita a dejar atrás el hegemónico paradigma adaptativo y reactivo que ha sostenido la interpretación del fenómeno educativo a modo de un sistema espejo de lo social.

En confluencia con lo anterior, los tradicionales modelos de FID participan de una profunda crisis (entendidas como condicionantes y posibilitadoras), debido al cuestionamiento de sus bases paradigmáticas y a la emergencia de nuevas formas de interpretación de los procesos de conocimiento y sus implicancias en el desarrollo multidimensional de los países. De este modo, el acentuado proceso de transformación epistemológico y antropo-social da forma a una reformulada noción de calidad educativa, basada en una gradual complejización (no trivialización) en su entendimiento. En este sentido, se da un tránsito desde modelos lineales y triviales referido a los procesos cognitivos, a concepciones que reconocen la complejidad del proceso educativo (CPEIP, MINEDUC, 2003), lo que a su vez redefine la profesión docente, alcanzando nuevos órdenes paradigmáticos en su interpretación y proyección.

No obstante, la complejidad de los procesos cognitivos, en estos términos, sigue desconociéndose bajo modelos simplistas e instrumentales, que se traducen en una serie de disyunciones de distinto orden, transversales a los procesos de FID en el país. El enfoque de la complejidad en este sentido no es sinónimo de complicación, sino de apertura a las posibilidades de convergencia e integración de diversas dimensiones aun profundamente desarticuladas en la FID, avocándose a las formas y pertinencia del conocimiento generado desde los espacios educativos.

Diversas investigaciones señalan la importancia de los docentes y su formación, no obstante, una serie de documentos y estudios en el ámbito internacional (Cochran-Smith, Zeichner y Fries, 2006; Vaillan, 2004) y nacional (Avalos, 2002) dan cuenta del estado deficitario de la FID. En el caso de Chile, el diagnóstico de la situación de la formación 
de profesores permite identificar algunos problemas en las formas de acción profesional, aun fuertemente mediadas por paradigmas basados en la linealidad, disyunción y descontextualización.

De este modo, se postula abordar un tema relativamente recurrente de investigación, pero desde una perspectiva epistemológica y metodológica diferente. Esto es, desde una dimensionalidad paradigmática compleja, destinada a indagar en las matrices semióticas que configuran la problemática educativa enunciada. A ello se añade la posibilidad de alcanzar un metacontexto de investigación, en el entendimiento que no es posible comprender y arribar a una solución de una problemática desde el mismo nivel epistémico en la cual fue generada.

La propuesta de la presente investigación radica en la posibilidad de indagar en estos procesos mediante una metodología cualitativa que integre elementos de orden cuantitativo. De este modo, el foco metodológico está en la caracterización y tipificación de nodos críticos de orden epistemológico, mediante la modelación de redes semióticas cogeneradas por estudiantes de diversos contextos de FID en Chile, con el objetivo de distinguir posibles atractores pedagógicos o centros semióticos de alta mediación sistémica (referirse al apartado de metodología) y sus consecuencias en las disyunciones formativas aun evidenciadas en el país.

De este modo, la posibilidad de generar estrategias comunicativas y formativas pertinentes y sostenibles está condicionada al desarrollo de una paradigmatología de lo educativo, como forma de entendimiento del sustrato semiótico donde se inscriben. Cabe señalar que es precisamente en esta última dimensión donde es posible significar las políticas educativas, por lo que el énfasis no sólo debería estar en lo que se debe implantar o introducir en el sistema, donde suele estar la atención de la dimensión política, sino además, en lo que los dominios paradigmáticos de dicho sistema le permiten interpretar y significar como pautas o esquemas de acción coherentes y plausibles.

Desde un enfoque epistemológico complejo, es fundamental una deconstrucción del conocimiento pedagógico para, desde allí, proyectar políticamente el sistema educativo en todos sus dominios. Así configurado el fenómeno, toda estrategia de acción comporta una dimensión cognitiva $\longrightarrow \longrightarrow$ comunicacional. (Morin, 1998: 65) por lo que es fundamental reconocer la relevancia de este dominio, tanto para el entendimiento de la problemática social, como para la teoría y acción política. (Tedesco, 2001) Tal como postula García Huidobro (2001), los procesos de transformación en educación son procesos esencialmente comunicativos, por lo que las estrategias de comunicación en este contexto no son posteriores a ellos, sino que los constituyen.

En este contexto, los problemas de la FID como una dimensión estrechamente vinculada a la calidad y a la equidad en los sistemas educativos vienen siendo observados desde la década del ochenta en todos los países del mundo (Bravslasky, 2002). En el caso de Chile, la evidencia empírica sobre la FID es consistente en la identificación de problemas centrales: formas inadecuadas de acción profesional, fragmentación de los contenidos disciplinarios y desconexión con los requerimientos pedagógicos. (Comisión sobre Formación Inicial, 2005; Díaz y Roa, 2009), lo que es congruente con la cotas de indagación y análisis de la presente propuesta. Asimismo, Cox (2004) afirma que en el proceso de reforma, dada su magnitud, convergen, entre otras dimensiones, la necesaria reformulación paradigmática implícita a toda innovación de las pautas de acción profesional y los serios problemas de desfase que muestra la formación de profesores. 
El amplio consenso sobre la importancia de la formación docente en las políticas de desarrollo ha dado origen a la discusión en torno a qué estrategias de innovación son pertinentes para las transformaciones educativas requeridas. En esta coyuntura se advierte que se ha perdido el sentido de esos procesos, quedando en segundo plano las estrategias orientadas en el cambio pedagógico (Tedesco, 1998). Tal como sugiere Freeman (1991), los procesos de FID deberían orientarse en hacer explícitos los sistemas epistemológicos implícitos al conocimiento profesional docente, mediante instancias de reflexión sobre su propia acción y desarrollo profesional.

La presente propuesta propone indagar los procesos de FID desde la base de tres dimensiones de análisis, caracterizadas por el estado del arte (MINEDUC, 2005; Avalos, 2005), como procesos aun profundamente disociados y por ello, nodos epistemológicos críticos en la FID en Chile. Dentro de las cotas de esta investigación, las dimensiones de análisis en torno a la construcción de conocimiento pedagógico refieren a los siguientes dominios de fractura cognitiva:

a) Desvinculación de los dominios de teoría/praxis.

b) Escisión de la formación pedagógica/disciplinar.

c) Incomunicación entre los diversos dominios disciplinares de formación pedagógica (condicionante para una educación intertransdisciplinaria).

Los dos primeros dominios ya han sido descritos ampliamente en el análisis realizado por la Comisión Nacional sobre FID en Chile (2005), el último es integrado por el equipo de investigación, desde el derrotero epistemológico complejo esbozado anteriormente, como un tercer orden de disyunción en FID. En consecuencia, la presente propuesta recoge esta plataforma de información cuantitativa y cualitativa, aportando nuevos elementos epistemológicos y recursos metodológicos en su interpretación.

En síntesis, las dimensiones de transformación e innovación que están a la base de la FID en el país son fenómenos no triviales, condicionados y complejizados por las matrices epistémicas y paradigmáticas que configuran sus estrategias e intencionalidades de acción en sus diferentes ámbitos.

\section{METODOLOGIA}

\section{ANTECEDENTES GENERALES}

El enfoque cualitativo de investigación es la base que sustentó esta investigación, aunque integró elementos técnicos de orden cuantitativo en el análisis de la información. Desde este enfoque, tal como sostiene Arnold (1998), comprendió una observación de segundo orden orientada a la observación de sistemas de observadores y sus respectivas observaciones. El núcleo de interés fue observar lo que los observados indican y describen, "el qué observan", como también, captar los esquemas de diferencias con que marcan tales observaciones y trazan sus distinciones, "el cómo observan". Lo anterior, desde un canon metodológico de orden sistémico. 


\section{FASE DE RECOLECCION DE INFORMACION}

a) Cotas comunicativas de la investigación: Estas quedaron definidas por los espacios temáticos pertinentes a la investigación, en este caso todos aquellos tópicos que generaron significado para los informantes en el contexto de su formación docente y configuración de conocimiento pedagógico, es decir, todo aquello cuya relación generó un significado para los sujetos en el contexto (ámbito o campo) comunicativo de los ejes de indagación definidos por la investigación: a) Desvinculación de los dominios de teoría/praxis, b) Escisión de la formación pedagógica/disciplinar, y c) Incomunicación entre los diversos dominios disciplinares de formación pedagógica.

b) Cotas estructurales de la investigación: En este caso, el campo de indagación consideró tres instancias de FID de universidades chilenas regionales definidas en sus directrices institucionales como universidades complejas y con al menos tres carreras de formación pedagógica ${ }^{1}$. Ello, dada las posibilidades que supone el concepto de universidad compleja para la formación docente, como asimismo, la evidencia que señala la baja prioridad que representan para este tipo de instituciones la formación de profesores (MINEDUC, 2005). El espacio social definido responde a las investigaciones de Villegas-Reimers (1998), Brunner, Elacqua, (2003) en las cuales se sostiene que la dimensión constitutiva al sistema educativo más importante en términos de calidad y equidad de la educación son los docentes y su formación.

c) Criterios de selección de participantes: Se consideró una etapa de diagnóstico y selección de informantes claves. La selección de casos para la investigación quedó definida en base a un muestreo estructural, el cual contempló a doce estudiantes de pedagogía de último año de formación de diversas áreas disciplinares de tres instituciones de educación superior del sur de Chile. Este criterio respondió a la necesidad de focalizar el estudio en etapas avanzadas de formación pedagógica con el objetivo de lograr una perspectiva integral de dichos procesos Las estrategias de recolección de información fueron atingentes a los procesos de formación propios de cada contexto, lo que se evaluó en la primera etapa de la investigación. Se consideró la realización de entrevistas en profundidad en todos los casos. Las técnicas de recolección de información consideradas respondieron a la necesidad de generar, en distintos niveles de análisis, eventos comunicativos regulados pero no directivos, que posibilitaron una aproximación a los procesos de configuración de conocimiento y sus dominios de identidad epistemológica.

\section{FASE DE ANALISIS DE INFORMACION}

a) Modelado de redes cognitivas: La técnica de análisis específica se basó en el concepto de redes cognitivas, esto es, un sistema computacional que grafica y cartografía los procesos asociativos y configuraciones (Lavanderos y Malpartida, 2000) en torno a la construcción de conocimiento, considerando la diversidad de elementos de sentido y los efectos sistémicos de su organización en dimensiones de sentido convergentes y divergentes.

1 El nombre de las instituciones específicas ha sido omitido por criterios de confidencialidad. 
b) Análisis de redes cognitivas: Desde la base de las redes semióticas modeladas, se desarrolló una batería de análisis de redes semióticas, entre los que se consideraron: análisis de conceptos iniciadores (tail) y conceptos terminales (head), análisis de dominio y centralidad semiótica (cálculo de atractores semióticos), conjunto jerárquico de agrupaciones (cluster) y análisis de ciclos tautológicos. El criterio para evaluar si existieron convergencias y/o divergencias entre los procesos de configuración de conocimiento pedagógico se focalizó en la conservación de los atractores de las redes generadas, estos es, en los nodos de mayor densidad relacional en términos semióticos (Lavanderos, Malpartida, 2000). Cabe enfatizar que la investigación no fue realizada sólo para rescatar las formas de conocimiento individuales de cada informante, sino además, como usuarios de los medios observacionales e interpretativos disponibles en el repertorio de sus contextos formativos. (Arnold, 1998).

En definitiva, la generación de redes cognitivas y la batería de análisis de información aplicados posibilitó tres niveles de indagación en términos de los procesos de configuración de conocimiento pedagógico: a) una caracterización de los elementos de inteligibilidad en torno a su construcción, b) las consecuencias de integración de dichos elementos en redes semióticas complejas y c) las inferencias a los contextos formativos-culturales en los que participan los estudiantes.

\section{SINTESIS INTERPRETATIVA DE RESULTADOS}

Los objetivos propuestos en el contexto del trabajo de campo de esta investigación refirieren a una cartografía a los procesos de configuración de conocimiento en el ámbito profesional docente, en profesores en formación de diversas instituciones de educación superior y pertenencia disciplinar. Ello, con el objetivo de indagar en tres nodos críticos o ejes de fractura cognitiva, a saber: desvinculación de los dominios de teoría/praxis, escisión de la formación pedagógica/disciplinar e incomunicación entre los diversos dominios disciplinares de formación pedagógica.

En este contexto, el siguiente apartado integra una síntesis interpretativa de los doce casos considerados en la investigación. La aproximación interpretativa debe entenderse a la luz de las redes construidas para cada caso, con el objetivo de no perder el contexto complejo y la trama relacional donde los códigos enfatizados fueron enunciados y configuraron sentido. La síntesis interpretativa se realizó desde los conceptos de mayor a menor grado de centralidad o mediación dentro de los planos de reformulación, considerando convergencias y divergencias entre los diversos informantes y contextos formativos.

En relación a las dimensiones de análisis propuestas en la investigación, la desvinculación de los dominios teoría y praxis pueden observarse en las vías argumentativas cartografiadas. Atractores tales como, necesidad de poner en práctica el conocimiento, manifiesta la necesidad de un conocimiento que sea pertinente al contexto educativo en el cual se desplegará profesionalmente. Esta última se presenta como un imperativo de la formación pedagógica y de la vinculación de los saberes en relación a los procesos de aprendizaje en entornos diversos de educación. Esta idea hace resonancia con otros atractores, la institución se preocupa mucho de la teoría en detrimento de la práctica y la práctica potencia el conocimiento teórico. 
La centralidad de estos atractores expresa una serie de falencias orientadas hacia los procesos de práctica, que desde una visión problematizada presentan divergencias entre la teorización excesiva de los contenidos y las competencias que no son vinculadas con la praxis profesional. Desde una visión operativa, los ejes o instancias de práctica para los estudiantes se presentan como un elemento de vital importancia en su formación desde los primeros años, ya que entrega un contexto situado desde donde proyectar competencias profesionales.

No obstante lo anterior, todos estos atractores mantienen un operador de disyunción implícito en la interpretación de la dialógica teoría-praxis. En estos términos, se reconoce la relevancia de la inmediatez y del conocimiento situado, pero con ello se mantiene y estabiliza una dimensión de fractura del fenómeno cognitivo, expresada en la secuencialización (y no simultaneidad) de los diversos niveles de recursión asociados a la cognición humana. Desde las cotas de esta investigación, el bucle conformado por la situacionalidad/inmediatez/sensibilidad y lo intencional/lógico/abstracto en FID, surge aun como un proceso fracturado en términos curriculares. En este sentido, la problemática no parece gestarse en la oscilación curricular entre la praxis o teoría en los programas de FID, sino más bien en su integración epistemológica.

A la vez, pudo observarse un significativo énfasis en los vínculos y pautas de convivencia en referencia a sus contextos de formación y práctica. En este nivel se hace latente la escisión entre la formación pedagógica y disciplinar, expresada en la necesidad de involucrarlas con la dimensionalidad compleja del fenómeno humano. En ello converge la necesidad de una educación que integre los diversos intereses, áreas de conocimiento, competencias teóricas, habilidades sociales y metodologías educativas. Los atractores la educación debe entenderse como fenómeno complejo y la disciplina por área se preocupa más de los contenidos que de la parte humana, hace una alusión particular a la excesiva especialización y desarticulación de los contenidos disciplinares, en detrimento de la concepción humana y compleja del conocimiento.

En este mismo dominio, también es posible caracterizar un desapego desde la voz de los estudiantes hacia la formación pedagógica; el atractor desvaloración de la educación en relación a la formación disciplinar caracteriza este fenómeno paradójico de la FID, en donde los futuros docentes expresan una relativa mayor valoración por la formación disciplinaria en contraste a la pedagógica. A la vez, aparece una percepción de desarticulación disciplinar/pedagógica, transversal a los diversos dominios disciplinares, derivada del insuficiente enlazamiento curricular en los programas de formación. Quizás ello refiera a una manifestación holográfica de las escisiones estructurales de las unidades académicas (facultades, departamentos y/o institutos) en los programas curriculares y en las dinámicas locales y globales en FID. Próximas investigaciones podrían indagar en el correlato epistemológico entre las dinámicas académicas de organización disciplinar y las disyunciones cognitivas de la FID en contextos específicos.

El lineamiento respecto al conocimiento generado en y para la praxis en contextos de desempeño sirve como referente a la dimensión de análisis en relación a la incomunicación entre los diversos dominios disciplinares de formación pedagógica. Los atractores involucramiento en áreas disciplinares distintas; la universidad requiere dar convergencia a las disciplinas; transversalidad de la enseñanza y entrelazamiento de contenidos, expresan el sentido y necesidad de organizar el conocimiento que se intenciona curricularmente de forma pertinente y atingente a las dinámicas complejas de 
generar y comunicar conocimiento en las sociedades modernas. Una solución tentativa a la problemática de la incomunicación de los campos disciplinares se expresa nuevamente en el nivel inmediato y situado (referida como nivel práctico), en el cual es posible hacer converger los diversos dominios disciplinares presentes en la formación docente. La falta de esta vinculación se manifiesta, en algunos casos, en una sensación de aletargamiento de las capacidades del estudiante para afrontar diversos problemas que no estén centrados en su área disciplinar particular. Asimismo, es expresada reiteradamente la necesidad de desarrollar competencias en distintos subsectores de forma transversal, como medio de generación de un conocimiento pertinente y significativo para los estudiantes.

En este contexto, es posible postular un sentido de necesidad por una educación inter y transdisciplinaria, no obstante, persisten rasgos de superficialidad en torno a la interpretación formal del diálogo entre disciplinas, como también una transversal jerarquización de contenidos que se proyecta en nuevas fracturas epistémicas entre las ciencias naturales y humanas. Estos resultados son en gran medida similares a los discutidos por Lerois (et al., 2007), en relación a la significación de la interdisciplinariedad en docentes en formación y ejercicio en el contexto internacional. Por último, la necesidad de integración y diálogo disciplinar presenta una amplia diversidad de interpretaciones y proyecciones en cada caso, las que no aparecen en las cotas de esta investigación, como un correlato a la pertenencia institucional ni campo disciplinar de los informantes.

\section{DISCUSIONES}

Derivado de lo anterior y en referencia a las convergencias de los diversos elementos de sentido interpretados en la investigación, es posible plantear que los tres ejes de fractura descritos en el marco referencial responden más a una lógica de simultaneidad que de secuencialidad. Esto último se expresa fenomenológicamente, más en una lógica de integración (mutua especificación sistémica de la problemática) que en la de paralelismo (dominios o ejes de fractura cognitiva de devenir independientes). En estos términos, las problemáticas epistemológicas asociadas a las fracturas y desarticulación de dominios formativos que sirvieron de base teórica a la presente investigación pueden responder a variantes superficiales de un mismo patrón paradigmático de disyunción, expresado holográficamente en distintos niveles y dimensiones de fractura en la formación de docentes en el país.

La necesidad de dar coherencia global e integración a los procesos de FID no pasa sólo por abordar e intervenir ejes de disyunción curricular de forma aislada a un nivel programático o metodológico, sino más bien reconocer la convergencia epistémica que subyace a ellos. Instancias de meta observación de la FID en el país pueden ayudar a cartografiar las matrices epistémicas y sociológicas que aún condicionan el diálogo entre instancias de teoría y praxis, formación pedagógica y disciplinar y por último, la comunicación inter y transdisciplinaria de los diversos campos disciplinares de formación pedagógica.

La integración multidimensional y compleja de los distintos niveles y regiones de formación, como asimismo la posibilidad de generar una visión ecológica, transdisciplinaria y compleja de la educación, está en riesgo bajo la actual congruencia de los sistemas curriculares formales de FID. Las fracturas cognitivas que persisten en estos procesos, en 
sus distintos niveles y dimensiones curriculares, impiden la asociación y reconstrucción de tejidos semióticos profundamente desgarrados y con ello restringen las condiciones de emergencia de nuevos estados de conciencia en el campo educativo, lo que en rigor superan ampliamente la noción de competencia profesional. Las cotas metodológicas de la presente investigación sólo proporcionan insumos cualitativos parciales a esta discusión, que necesariamente deberán ser complementados con otras ópticas metodológicas y epistemológicas.

\section{BIBLIOGRAFIA}

Arnold, M. (1998). Recursos para la investigación sistémico/constructivista. Facultad de Ciencias Sociales. Universidad de Chile. Cinta de Moebio No 3. Chile. Disponible en www.moebio. uchile.cl (Visitado septiembre 2003).

Arnold, M. y D. Rodríguez (1999). Sociedad y Teoría de sistemas. Ed. Universitaria. Santiago de Chile.

Avalos, B. (2002). Profesores para Chile, Historia de un proyecto. MINEDUC. Santiago de Chile.

Avalos, B. (2005). La formación de profesores y su desarrollo profesional. En Cox (Edit.), Políticas educacionales en el cambio de siglo. Cap. XI, pp. 559-598. Universitaria. Santiago.

Bravslasky, C. (2002). Desarrollo de las propuestas de formación docente inicial frente a los desafios de la mundialización. Simposio Internacional: Perspectivas de formación docente. Perú.

Brunner, J. y G. Elacqua (2003). Informe capital humano en Chile. Universidad Adolfo Ibáñez. Escuela de Gobierno. Santiago de Chile.

Cochran-Smith, M., K. Zeichner y K. Fries (2006). Estudio sobre la formación del profesorado en los Estados Unidos. Revista de Educación, № 1, pp. 87-116.

Comisión sobre Formación Inicial (2005). Informe Comisión sobre formación inicial docente. MINEDUC. Santiago.

Cornejo, J. (1999). Profesores que se inician en la docencia: Algunas reflexiones al respecto desde América Latina. Revista Iberoamericana de Educación (OEI), 1999, № 19: 51-100.

Cox, C. (2004). Las políticas educacionales en Chile en las últimas dos décadas del siglo XX. En Cox (Edit.), Políticas educacionales en el cambio de siglo. Cap. I., pp. 19-114. Universitaria. Santiago.

CPEIP, Ministerio de Educación de Chile (2003). Marco para la buena enseñanza. C\&C. Santiago de Chile.

Díaz, C. e I. Roa (2009). Las concepciones pedagógicas de estudiantes en práctica profesional respecto de su rol como profesores. Boletín de Investigación Educacional, Vol. 24, pp. 183-194.

Freeman, D. (1991). To make the tacit explicit: Teacher education, emerging discourse, and conceptions of teaching. Teaching and Teacher Education, 7 (5/6), pp. 439-454.

García-Huidobro J. y C. Cox (1999). La Reforma Educacional chilena. Editorial Popular, Madrid, España.

García Huidobro, J. (2001). Conflictos y alianzas en las reformas educativas. Siete tesis basadas en la experiencia chilena. En Martinic y Prado (editores), Economía y política de las reformas educativas en América Latina. Informe CIDE-PREAL, Santiago de Chile.

Lavanderos, L. y A. Malpartida (2000). Tópicos en torno a la cognición relacional. Centro de Estudios en Teoría Relacional y Sistemas de Conocimiento. Santiago de Chile.

Lenoir, Y., A. Hasni et F. Larose (2007). L'interdisciplinarité et la formation à l'enseignement: analyse de résultats de deux recherches. Revista Pensamiento Educativo, Vol. 41., $\mathrm{N}^{\circ} 2$. pp. 255-276. 
Ministerio de Educación de Chile (1998). Reforma en marcha: Buena educación para todos. Documento oficial. Santiago de Chile.

Morin, E. (1998). El método: La vida de la vida. Ediciones Cátedra. Madrid.

Morin, E. (1999). La cabeza bien puesta: Repensar la reforma $\longrightarrow$ Reformar el pensamiento. Nueva Visión. Buenos Aires.

OCDE (2009). Revisión de Políticas nacionales de Educación. La educación superior en Chile. MINEDUC. Santiago.

Tedesco, J. C. (1998). Desafíos de las Reformas Educativas en América Latina. IIPE. Buenos Aires.

Tedesco, J. C. (2001). Desafíos políticos de las reformas de la educación. En Martinic y Prado (editores), Economía y política de las reformas educativas en América Latina. Informe CIDEPREAL, Santiago de Chile.

Vaillant, D. (2002). Formación de formadores. Estado de la práctica. Preal. Editorial San Marino, Santiago de Chile.

Villegas-Reimers, E. (1998). The preparation of teachers in Latin America. The World Bank, Human Development Department. 


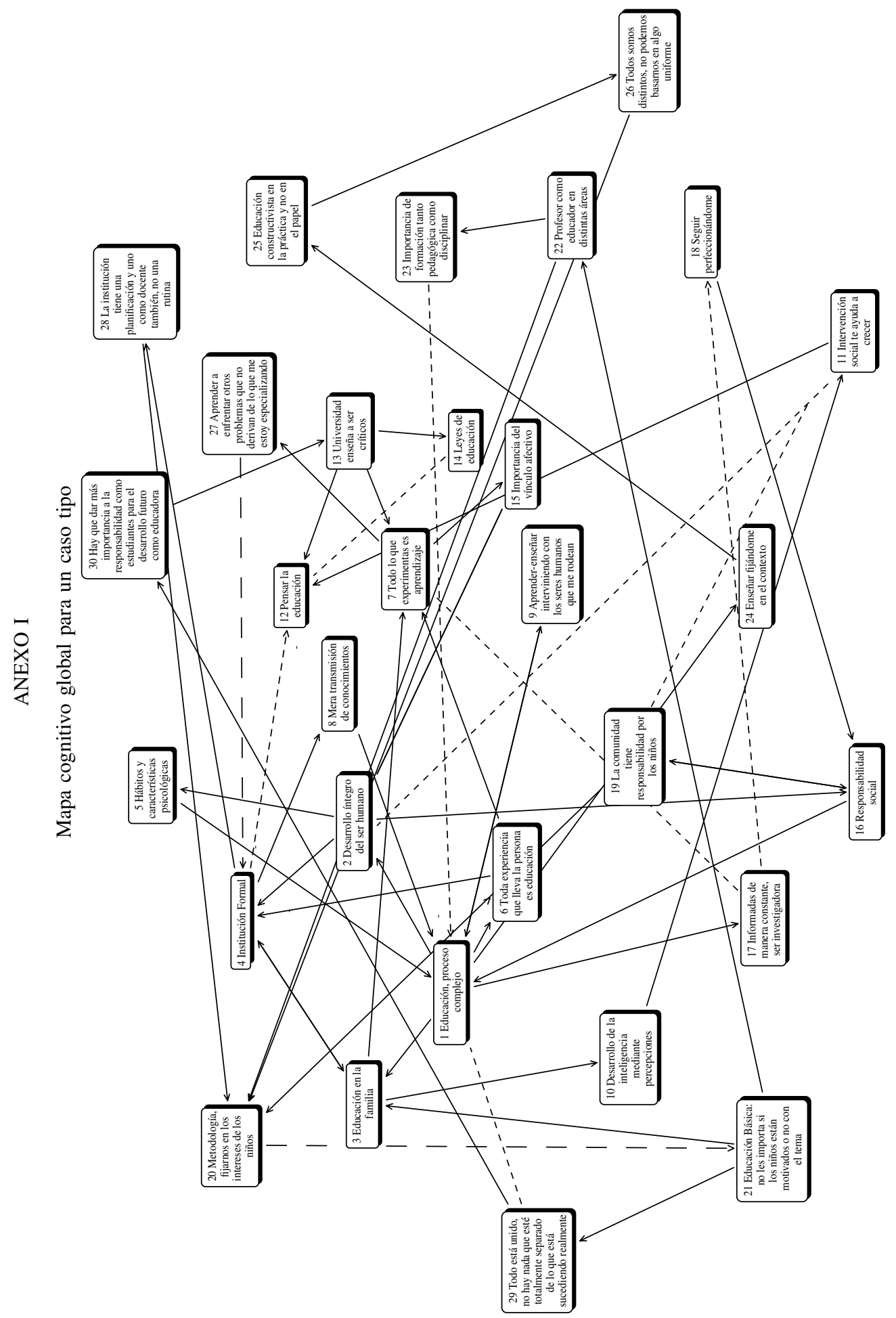




\section{ANEXO II}

Desglose de los tres atractores principales para un caso tipo
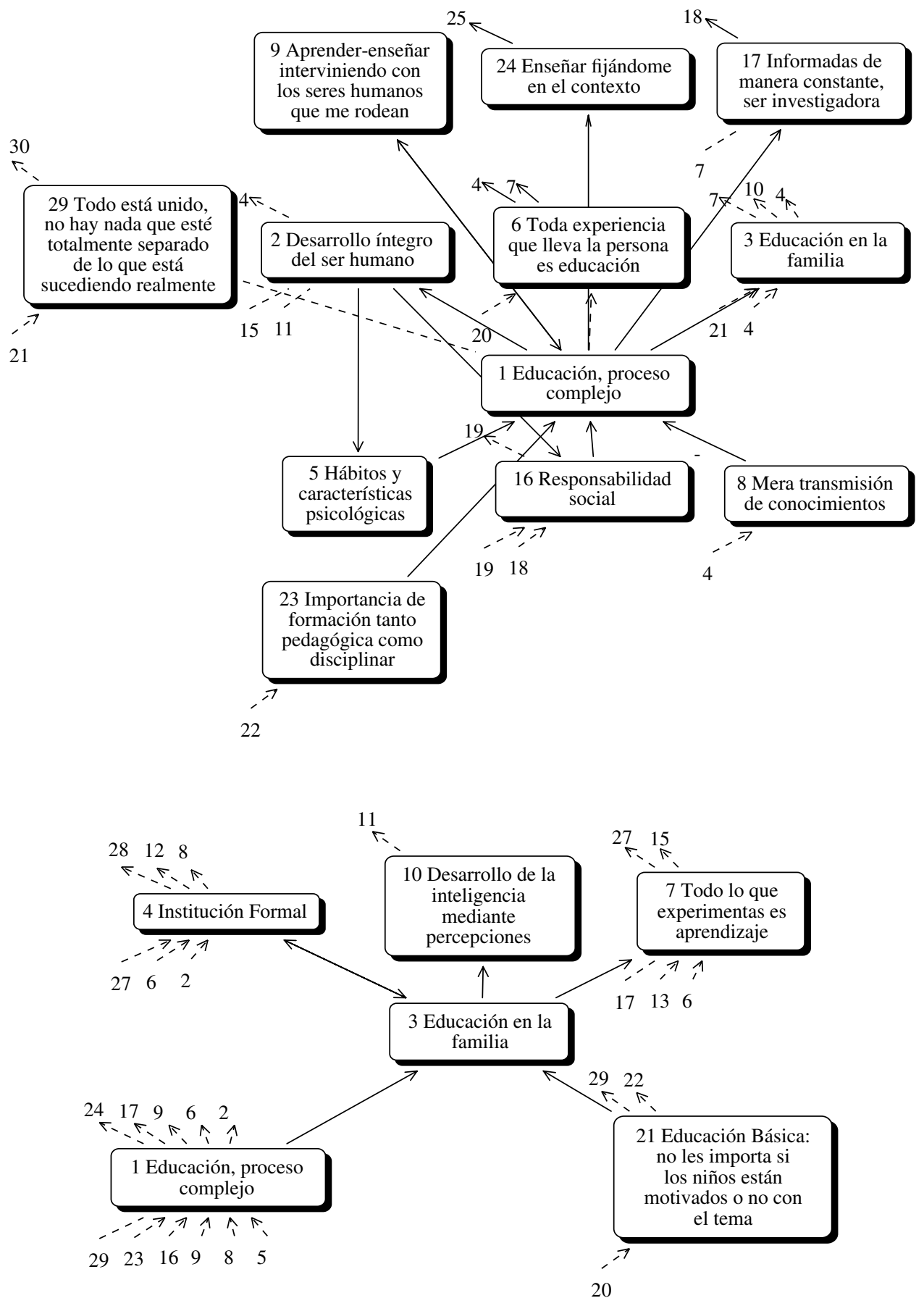
(continuación Anexo II)

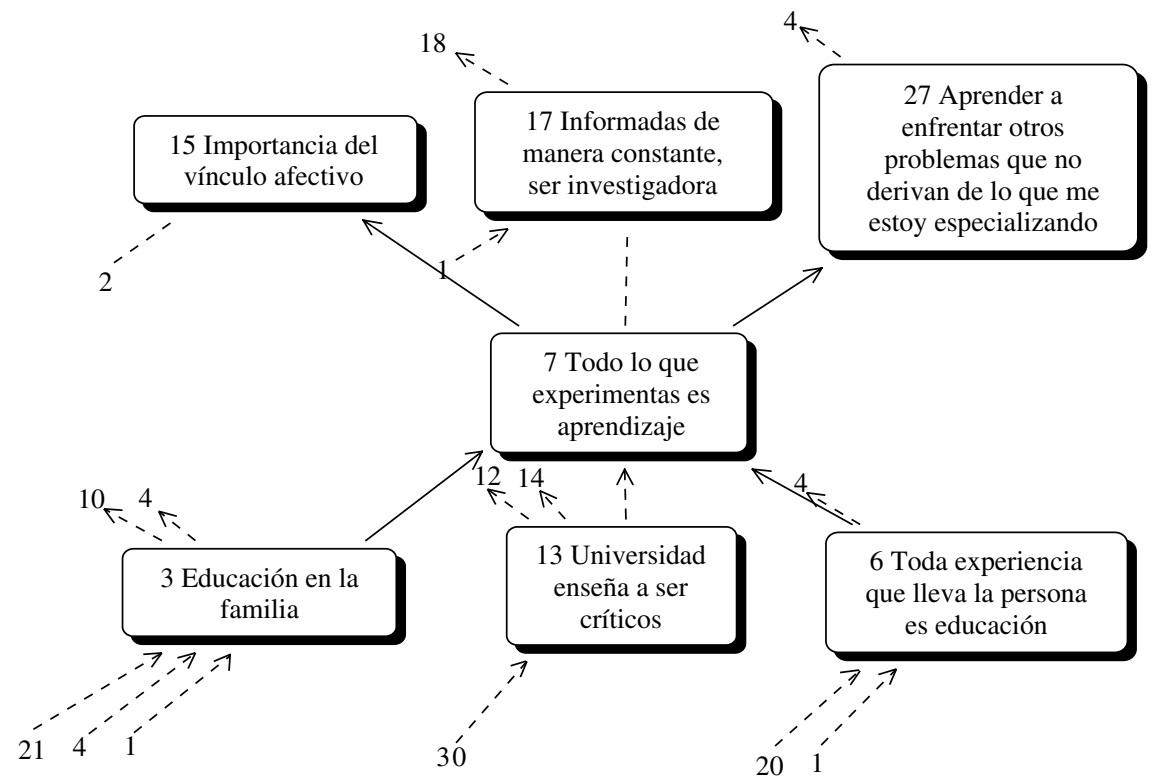


